\title{
OPTIMAL PATHS ON THE SPACE-TIME SINR RANDOM GRAPH
}

\author{
FRANÇOIS BACCELLI, ${ }^{*}$ INRIA/ENS \\ BARTŁOMIEJ BŁASZCZYSZYN, ${ }^{* *}$ INRIA/ENS and Math. Inst. University of Wroctaw \\ MIR OMID HAJI MIRSADEGHI, ${ }^{* * *}$ INRIA/ENS and Sharif University of Technology
}

\begin{abstract}
We analyze a class of Signal-to-Interference-and-Noise-Ratio (SINR) random graphs. These random graphs arise in the modeling packet transmissions in wireless networks. In contrast to previous studies on the SINR graphs, we consider both a space and a time dimension. The spatial aspect originates from the random locations of the network nodes in the Euclidean plane. The time aspect stems from the random transmission policy followed by each network node and from the time variations of the wireless channel characteristics. The combination of these random space and time aspects leads to fluctuations of the SINR experienced by the wireless channels, which in turn determine the progression of packets in space and time in such a network.

This paper studies optimal paths in such wireless networks in terms of first passage percolation on this random graph. We establish both "positive" and "negative" results on the associated time constant. The latter determines the asymptotics of the minimum delay required by a packet to progress from a source node to a destination node when the Euclidean distance between the two tends to infinity. The main negative result states that this time constant is infinite on the random graph associated with a Poisson point process under natural assumptions on the wireless channels. The main positive result states that when adding a periodic node infrastructure of arbitrarily small intensity to the Poisson point process, the time constant is positive and finite.
\end{abstract}

Keywords: Poisson point process, random graph, first passage percolation, shotnoise process, SINR

\footnotetext{
* Postal address: ENS DI TREC, 45 rue d'ulm, 75230 Paris, FRANCE.

** Postal address: ENS DI TREC, 45 rue d'ulm, 75230 Paris, FRANCE.

*** Postal address: ENS DI TREC, 45 rue d'ulm, 75230 Paris, FRANCE. The work of this author is part of a joint PhD programme with a co-advising by Prof. Amir Daneshgar in Tehran
} 
2000 Mathematics Subject Classification: Primary 60D05, 05C80

Secondary 90C27, 60G55

\section{Introduction}

There is a rich literature on random graphs generated over a random point process. These graphs are often motivated by physical, biological or social networks. Many interesting large scale properties of these networks related to connectivity have been studied in terms of the percolation of the associated graphs. An early example of such a study can be found in [13] where the connectivity of large networks was defined as the supercritical phase in what is today called the continuum (Boolean) percolation model. More recently, a random SINR graph model for wireless networks was studied with the same perspective in $[10,11]$.

The routing, and more precisely, the speed of delivery of information in networks is another example of problems, which motivated the study of the random graphs. The main object in this context is the evaluation of the so called time constant, which gives the asymptotic behavior of the number of edges (hops) in the paths (optimal or produced by some particular routing protocol) joining two given nodes in function of the (Euclidean) distance between these nodes, when this distance tends to infinity. In the case of a shortest (in terms of the number of hops) path, this problem is usually called the first passage percolation problem and was originally stated by Broadbent and Hammersley in [8] to study the spread of fluid in a porous medium. More recently, in $[20,7]$, such time constants were studied on so called small word graphs, motivated by routing in certain social networks, where any two given nodes are joined by an edge independently with a probability that decays as some power function with the Euclidean distance between them. The complete graph on a Poisson p.p. with "nearest neighbor" routing policy was studied in this context in [6]. The first passage percolation problem on the Poisson-Delaunay graph was considered in [24, 21]. In the case of graphs whose edges are marked by some weights, one can extend the notion of time constant by studying the sum of the edge weights. First passage percolation on the complete Poisson p.p. graph, with weights proportional to some power of the distance between the nodes was studied in [14].

The present paper focuses on the speed of delivery of information in SINR graphs. In 
contrast to previous studies of this subject, in particular to [10,11], we consider graphs with space-time vertexes. This new model is motivated by multihop routing protocols used in wireless ad-hoc networks. In this framework, the random point process on the plane describes the locations of the users of an ad-hoc network and the discrete time dimension corresponds to successive time slots in which these nodes exchange information (here packets). As in [2] we assume the spatial Aloha policy to decide which node transmit at a given time slot. We also assume some space-time fading model (already used e.g. in [5]) to describe the variability of the wireless channel conditions (see e.g. [23]). In this space-time SINR graph, a directed edge represents the feasibility of the wireless transmission between two given network nodes at a given time. More precisely, the direct transmission of a packet is succeeds between two nodes in a given time slot if the ratio of the power of the signal between these nodes to the interference and noise at the receiver is larger than a threshold at this time slot. This definition has an information theoretic basis (see e.g. [23]). It is rigorously defined below using some power path-loss model and an associated shot-noise model representing the interference.

We study various problems on this random graph including the law of its in- and out-degree, the number of paths originating from (or terminating at) a typical node or its connectedness. The most important results bear on the first passage percolation problem in this graph. In the case of Poisson p.p. for the node locations, we show that the time constant is infinite. We then show that when adding a periodic node infrastructure of arbitrarily small intensity to the Poisson point process, the time constant is positive and finite. These results lead to bounds on the delays in ad-hoc networks which hold for all routing algorithms. This subject, or more generally, the question of the speed of the delivery of information in large wireless ad-hoc networks currently receives a lot of attention in the engineering literature see e.g. [12, 15].

The paper is organized as follows. In Section 2 we introduce the space-time SINR graph model. The results are presented in Section 3, Most of the proofs are deferred to Section 4. Some implications on routing in ad-hoc networks are presented in Section 5. 


\section{The Model}

\subsection{Probabilistic Assumptions}

Throughout the paper we consider a simple, stationary, independently marked (i.m.)

point process (p.p.) $\widetilde{\Phi}=\left\{\left(X_{i}, \mathbf{e}_{i}, \mathbf{F}_{i}, \mathbf{W}_{i}\right)\right\}$ with finite, positive intensity $\lambda$ on $\mathbb{R}^{2}$. In this model,

- $\Phi=\left\{X_{i}\right\}$ denotes the locations of the network nodes on the plane $\mathbb{R}^{2}$. The following three cases regarding the distribution of $\Phi$ will be considered:

General p.p.: $\Phi$ is a general (stationary, non-null, with finite intensity) p.p.,

Poisson p.p.: $\Phi$ is a Poisson p.p.,

Poisson+Grid p.p.: $\Phi=\Phi_{M}+\Phi_{G}$ is the superposition of two independent p.p.s; where $\Phi_{M}$ denotes a stationary Poisson p.p. with finite, non-null intensity $\lambda_{M}$ and $\Phi_{G}=s \mathbb{Z}^{2}+U_{G}$ a stationary, periodic p.p., whose nodes constitute a square grid with edge length $s$, randomly shifted by the vector $U_{G}$ that is uniformly distributed in $[0, s]^{2}$ (this makes $\Phi_{G}$ stationary). Note that the intensity of $\Phi_{G}$ is $\lambda_{G}=1 / s^{2}$.


$\left\{e_{i}(n): i, n\right\}$ are i.i.d. (in $n$ and $i$ ) Bernoulli random variables (r.v.s) with $\mathbf{P}\{e=1\}=1-\mathbf{P}\{e=0\}=p$, where $e$ denotes the generic r.v. for this family. We always assume $0<p<1$. The variable $e_{i}(n)$ represents the medium access indicator of node $X_{i}$ at time $n$; it says whether the node transmits (case $e=1$ ) or not at time $n$.

- $\mathbf{W}_{i}=\left\{W_{i}(n)\right\}_{n} ;\left\{W_{i}(n): i, n\right\}$ is a family of non-negative i.i.d. r.v.s with some arbitrary distribution. The variable $W_{i}(n)$ represents the power of the thermal noise at node $X_{i}$ and at time $n$. Let $W$ denote the generic r.v. for this family.

- $\mathbf{F}_{i}=\left\{F_{i, j}(n)\right\}_{j, n} ;\left\{F_{i, j}(n): i, j, n\right\}$ is a family of non-negative i.i.d. r.v.s. The variable $F_{i, j}(n)$ represents the quality of the radio channel (also called fading) from node $X_{i} \in \Phi$ to node $X_{j} \in \Phi$ at time $n$. The following two cases regarding the distribution of $F$ (denoting the generic random variable for this family) will be considered: 
General fading: when $F$ has some arbitrary distribution with finite mean.

Exponential fading: when $F$ has exponential distribution with mean $1 / \mu$. $\left(^{*}\right)$

To complete the probabilistic description of the model we assume that, given $\Phi$, the random elements $\left\{\mathbf{e}_{i}\right\}_{i},\left\{\mathbf{W}_{i}\right\}_{i}$ and $\left\{\mathbf{F}_{i}\right\}_{i}$ are independent. For more on this framework, which is classical, see e.g. or $[3,4,5]$.

Our stationary i.m. p.p. $\tilde{\Phi}$ is considered on some probability space with probability $\mathbf{P}$. We will denote by $\mathbf{P}^{0}$ the Palm probability with respect to $\Phi$; see [9, Ch.13]. Recall that it can be interpreted as the conditional probability given $\Phi$ has a point at the origin 0 of the plane. We will denote this point (considered under $\mathbf{P}^{0}$ ) by $X_{0}$ and call it the typical node. Under $\mathbf{P}^{0} \tilde{\Phi}$ is also an i.m. p.p. with marks distributed as in the original law. Moreover, in the case of Poisson p.p.s, the distribution of $\Phi$ under $\mathbf{P}^{0}$ is equal to the distribution of $\Phi \cup\left\{X_{0}=0\right\}$ under the stationary probability $\mathbf{P}$ (cf. the Slivnyak-Mecke Theorem [9, p.281]).

\subsection{SINR Marks}

Given the i.m.p.p. $\tilde{\Phi}$ described above, we construct another family of random variables $\left\{\operatorname{SINR}_{i j}(n): i, j, n\right\}$, which will be interpreted as the SINR observed in the channel form $X_{i} \in \Phi$ to $X_{j} \in \Phi$ at time $n$. These variables, which have an information theoretic background, will be used to assess the success of transmissions. For defining these variables, we give ourselves some non-decreasing function $l: \mathbb{R}^{+}=\{t: t \geq 0\} \rightarrow$ $\mathbb{R}^{+}$that we call the path-loss function. A special example considered in this paper (and commonly accepted in the wireless communication context) is

$$
l(r)=(A r)^{\beta} \quad \text { with some } A>0 \text { and } \beta>2 .
$$

Denote by $\Phi^{1}(n)=\left\{X_{i}: e_{i}(n)=1\right\}$ the point process of transmitters at time slot $n$ and by $\Phi^{0}(n)=\left\{X_{i}: e_{i}(n)=0\right\}$ that of (potential) receivers. For a given pair receiver $X_{j} \in \Phi^{0}$ and transmitter $X_{i} \in \Phi^{1}(n)$, we will assume that $X_{j}$ receives a signal from $X_{i}$ with power $F_{i, j}(n) / l\left(\left|X_{j}-X_{i}\right|\right)$ at time $n$. Node $X_{j}$ also receives signals from other

(*) In wireless signal propagation models, the exponential distribution appears naturally as the square power of the norm of a complex random variable, whose real and imaginary components are i.i.d. Gaussian. I this case one often speaks about Rayleigh fading model because the norm (absolute value) of such a complex random variable is Rayleigh-distributed; see e.g. [23, p. 50 and 501]. 
transmitters $X_{k} \in \Phi^{1}(n), X_{k} \neq X_{i}$ at time $n$. The total received power is equal to

$$
I_{i, j}(n)=\sum_{X_{k} \in \Phi^{1}(n) \backslash\left\{X_{i}\right\}} F_{k, j}(n) / l\left(\left|X_{k}-X_{j}\right|\right) .
$$

Let also

$$
I_{j}(n)=\sum_{X_{k} \in \Phi^{1}(n) \backslash\left\{X_{j}\right\}} F_{k, j}(n) / l\left(\left|X_{k}-X_{j}\right|\right) .
$$

Both $I_{i, j}(n)$ and $I_{j}(n)$ are shot-noise r.v.s generated by $\Phi^{1}(n)$, the fading marks and the path-loss function. The are infinite sums of non-negative r.v.s. In order to check whether these r.v.s are a.s. finite, one can use the Campbell-Little-Mecke formula (Campbell for short; cf. [9, Prop. 13.3.II]), which implies that

$$
\mathbf{E}^{0}\left[\sum_{X_{k} \in \Phi^{1}(n),\left|X_{k}\right|>\epsilon} F_{k, 0}(n) / l\left(\left|X_{k}\right|\right)\right]=p \mathbf{E}[F] \int_{\mathbb{R}^{2} \backslash[0, \epsilon]^{2}} 1 / l(|x|) \breve{M}_{[2]}(\mathrm{d} x),
$$

where $\breve{M}_{2}(\cdot)$ is the reduced second order moment measure of $\Phi$ (cf [9, p. 238]). In what follows, we will always tacitly assume that $l(\cdot), \Phi$ are such that the integral in the right-hand side of (2) is finite for some $\epsilon \geq 0$, which implies that $I_{0}(n)$ is almost surely (a.s.) finite under $\mathbf{P}^{0}$ for all $n$ as well as all $I_{j}(n), I_{i, j}(n)$ under $\mathbf{P}$. If $\Phi$ is the homogeneous Poisson p.p., we have $\breve{M}_{[2]}(\mathrm{d} x)=\lambda \mathrm{d} x$ and it is easy see that the we have finiteness for $l(\cdot)$ given by (1) for all $\epsilon>0$. It is also relatively easy to see that it holds for the Poisson+Grid p.p. $\Phi=\Phi_{M}+\Phi_{G}$.

The SINR at the receiver $X_{j} \in \Phi^{0}(n)$ with respect to transmitter $X_{i} \in \Phi^{1}(n)$, at time $n$ is defined as

$$
\operatorname{SINR}_{i, j}(n)=\frac{F_{i, j}(n) / l\left(\left|X_{i}-X_{j}\right|\right)}{W_{j}(n)+I_{i, j}(n)}
$$

\subsection{Space-Time SINR Graph}

Let

$$
\delta_{i, j}(n)= \begin{cases}\mathbb{1}\left(\operatorname{SINR}_{i, j} \geq T\right) & \text { if } e_{i}(n)=1, e_{j}(n)=0, i \neq j \\ 1 & \text { if } i=j, \\ 0 & \text { otherwise }\end{cases}
$$


where $T>0$ is a threshold assumed to be some given constant throughout the paper. We define the space-time SINR graph $\mathbb{G}$ as the directed graph with the set of vertexes $\Phi \times \mathbb{Z}$ and a directed edge from $\left(X_{i}, n\right)$ to $\left(X_{j}, n+1\right)$ if $\delta_{i, j}(n)=1$.

Let us stress an important convention in our terminology. By network node, or point, we understand a point of $\Phi$. A (graph) vertex is an element of $\Phi \times \mathbb{Z}$; i.e. it represents some network node at some time. The existence of a graph edge is to be interpreted as the possibility of a successful communication between two network nodes (those involved in the edge) at time $n$. This can be rephrased as follows. Suppose that at time $n$ the network node $X_{i}$ has a packet (containing some information). Then the set of graph neighbors of the vertex $\left(X_{i}, n\right)$ describes all the nodes that can decode this packet at time $n+1$. Thus any path on the graph $\mathbb{G}$ represents some possible route of the packet in space and time.

\section{Results}

In this section we present our results on $\mathbb{G}$.

\subsection{Existence of Paths}

All the results of this section are obtained under the general p.p. and fading assumptions of Section 2, under the assumption that the finiteness of the expression in (2) is granted.

Note first that $\mathbb{G}$ has no isolated nodes in the usual sense. Indeed, we have always $\left(X_{i}, n\right)$ connected to $\left(X_{i}, n+1\right)$. We will consider directed paths on $\mathbb{G}$ and call them paths for short. Note that these paths are self-avoiding due to the fact that there are no loops in the time dimension.

Denote by $\mathcal{H}_{i}^{\text {out }, k}(n)$ the number of paths of length $k$ (i.e. with $k$ edges) originating from $\left(X_{i}, n\right)$. Similarly, denote by $\mathcal{H}_{i}^{i n, k}(n)$ the number of such path terminating at $\left(X_{i}, n\right)$. In particular $\mathcal{H}_{i}^{\text {out }}(n)=\mathcal{H}_{i}^{\text {out }, 1}(n)$ and $\mathcal{H}_{i}^{\text {in }}(n)=\mathcal{H}_{i}^{\text {in, },}(n)$ are respectively, the out- and in-degree of the node $\left(X_{i}, n\right)$.

Lemma 1. For a general p.p. $\Phi$ and a general fading model, the in-degree $\mathcal{H}_{i}^{i n}$ of any node of $\mathbb{G}$ is bounded from above by the constant $\xi=1 / T+2$.

Proof. Assume there is an edge to node $\left(X_{j}, n\right)$ from nodes $\left(X_{i_{1}}, n-1\right), \ldots,\left(X_{i_{k}}, n-\right.$ 
$1)$, for some $k>1$ and $i_{p} \neq j(p=1, \ldots, k)$. Then for all such $p$

$$
\frac{F_{i_{p}, j}}{l\left(\left|X_{i_{p}}-X_{j}\right|\right)} \geq \frac{T}{1+T}\left(\sum_{q=1}^{k} \frac{F_{i_{q}, j}}{l\left(\left|X_{i_{q}}-X_{j}\right|\right)}\right)
$$

When summing up all these inequalities, one gets that $T k \leq 1+T$, that is $k \leq 1 / T+1$. Considering the edge from $\left(X_{i}, n-1\right)$ to $\left(X_{i}, n\right)$, the in-degree of any node is bounded from above by $\xi=1 / T+2$.

Let

$$
h^{\text {out }, k}=\mathbf{E}^{0}\left[\mathcal{H}_{0}^{\text {out }, k}(n)\right]=\mathbf{E}^{0}\left[\mathcal{H}_{0}^{\text {out }, k}(0)\right]
$$

and

$$
h^{i n, k}=\mathbf{E}^{0}\left[\mathcal{H}_{0}^{i n, k}(n)\right]=\mathbf{E}^{0}\left[\mathcal{H}_{0}^{i n, k}(0)\right]
$$

be the expected numbers of paths of length $k$ originating or terminating at the typical node, respectively. In particular $h^{\text {out }}=h^{\text {out }, 1}$ and $h^{i n}=h^{i n, 1}$ are the mean out- and in-degree of the typical node, respectively.

Lemma 2. For a general p.p. $\Phi$ and a general fading model

$$
h^{\text {in }, k}=h^{\text {out }, k} \text {. }
$$

Proof. We use the mass transport principle to get that $\mathbf{E}^{0}\left[\mathcal{H}_{0}^{\text {out }, k}(0)\right]=\mathbf{E}^{0}\left[\mathcal{H}_{0}^{\text {in,k }}(0)\right]$, which implies the desired result. Indeed, Campbell's formula and stationarity give

$$
\begin{aligned}
\lambda h^{\text {out }, k} & =\lambda \int_{[0,1)^{2}} \mathbf{E}^{0}\left[\mathcal{H}_{0}^{\text {out }, k}(0)\right] \mathrm{d} x \\
& =\mathbf{E}\left[\sum_{X_{i} \in \Phi \cap[0,1)^{2}} \mathcal{H}_{i}^{\text {out }, k}(0)\right] \\
& =\sum_{v \in \mathbb{Z}} \mathbf{E}\left[\sum_{X_{i} \in[0,1)^{2}} \sum_{X_{j} \in[0,1)^{2}+v} \# \text { of paths from }\left(X_{i}, 0\right) \text { to }\left(X_{j}, k\right)\right] \\
& =\sum_{v \in \mathbb{Z}} \mathbf{E}\left[\sum_{X_{i} \in[0,1)^{2}-v} \sum_{X_{j} \in[0,1)^{2}} \# \text { of paths from }\left(X_{i}, 0\right) \text { to }\left(X_{j}, k\right)\right] \\
& =\lambda \int_{[0,1)^{2}} \mathbf{E}^{0}\left[\mathcal{H}_{0}^{\text {in }, k}(k)\right] \mathrm{d} x=\lambda h^{i n, k},
\end{aligned}
$$

where \# denotes the cardinality. This completes the proof. 
Here are immediate consequences of the two above lemmas.

Corollary 3.1. Under the assumptions of Lemma 1:

- $\mathbb{G}$ is locally finite (both on in-and out-degrees of all nodes are $\mathbf{P}$-a.s. finite).

- $\mathcal{H}_{i}^{i n, k}(n) \leq \xi^{k} \mathbf{P}$-a.s for all $i, n, k$.

- $h^{\text {in,k }}=h^{\text {out }, k} \leq \xi^{k}$ for all $k$.

For all $X_{i}, X_{j} \in \Phi$ and $n \in \mathbb{Z}$, we denote by we will call local delay from $X_{i}$ to $X_{j}$ at time $n$ the quantity

$$
L_{i, j}(n)=\inf \left\{k \geq n: \delta_{i, j}(k)=1\right\}
$$

with the usual convention that $\inf \emptyset=\infty$. Note that $L_{i, j}(n)$ is the length (number of edges) of the shortest path (with the smallest number of edges) from $\left(X_{i}, n\right)$ to $\left\{X_{j}\right\} \times \mathbb{Z}$ among the paths contained in the subgraph $\mathbb{G} \cap\left\{X_{i}, X_{j}\right\} \times \mathbb{Z}$ of $\mathbb{G}$, which is of the form

$$
\begin{aligned}
& \left(\left(X_{i}, n\right),\left(X_{i}, n+1\right)\right), \ldots,\left(\left(X_{i}, n+L_{i, j}(n)-1\right),\left(X_{i}, n+L_{i, j}(n)\right)\right), \\
& \left(\left(X_{i}, n+L_{i, j}(n)\right),\left(X_{j}, n+L_{i, j}(n)+1\right)\right) .
\end{aligned}
$$

Our next result gives a condition for the local delays to be a.s. finite.

Lemma 3. Assume a general p.p. $\Phi$ and a general fading model with $F$ having unbounded support $(\mathbf{P}\{F>s\}>0$ for all $0<s<\infty)$. Then, given $\Phi$, all local delays $L_{i, j}(n)$ are $\mathbf{P}$-a.s. finite geometric random variables.

Proof. Due to our assumption on the independence of marks in successive time slots, given $\Phi$, the variables $\left\{\delta_{i, j}(n): n \in \mathbb{Z}\right\}$ are (i.i.d.) Bernoulli r.v. and thus $L_{i, j}(n)$ is geometric r.v. It remains to show that $\mathbf{P}\left\{\delta_{i, j}(0)=1 \mid \Phi\right\}:=\pi_{i, j}(\Phi)>0$ for $\mathbf{P}$-almost all $\Phi$. For this, note that

$$
\pi_{i, j}(\Phi)=p(1-p) \mathbf{P}\left\{F_{i, j}(0) \geq l\left(\left|X_{j}-X_{i}\right|\right)\left(W_{j}(0)+I_{i, j}(0)\right)\right\}
$$

Under our general assumptions (including finiteness of the expression in (2)) $I_{i, j}(0)$ is a finite random variable $\mathbf{P}$-a.s. The result follows from the assumption that $0<p<1$ 
and the fact that $F_{i, j}(0)$ is independent of $I_{i, j}(0), W_{i, j}(0)$ and has infinite support.

The next result directly follows from Lemma 3.

Corollary 3.2. Under the assumptions of Lemma 3, $\mathbb{G}$ is $\mathbf{P}$-a.s. connected in the following weak sense: for all $X_{i}, X_{j} \in \Phi$ and all $n \in \mathbb{Z}$, there exists a path from $\left(X_{i}, n\right)$ to the set $\left\{\left(X_{j}, n+l\right): l \in \mathbb{N}\right\}$, where $\mathbb{N}=\{1,2, \ldots\}$.

We denote by $L_{i}(n)=\inf _{j \neq i} L_{i, j}$ the length of a shortest directed path from $\left(X_{i}, n\right)$ to $\left(\left\{\Phi \backslash X_{i}\right\}\right) \times \mathbb{Z}$. We will call $L_{i}(n)$ the exit delay from $X_{i}$ at time $n$. Finally, we denote by $P_{i, j}(n)$ the length of a shortest path of $\mathbb{G}$ from $\left(X_{i}, n\right)$ to $\left\{X_{j}\right\} \times \mathbb{Z}$. We call $P_{i, j}(n)$ the delay from $X_{i}$ to $X_{j}$ at time $n$. Obviously for $i \neq j$ we have

$$
L_{i}(n) \leq P_{i, j}(n) \leq L_{i, j}(n)
$$

and thus it follows immediately from Lemma 3 that all the three collections of delays finite r.v.s $\mathbf{P}$-a.s.

\subsection{Optimal Paths - Poisson p.p. Case}

We have seen in the previous section that under very general assumptions, all the delays are $\mathbf{P}$-a.s. finite random variables. In this section we show that under some natural assumptions (such as Poisson p.p. and exponential fading), the averaging over $\Phi$ may lead to infinite mean values. This averaging is expressed in terms of the expectation for the typical node under the Palm probability. The proofs of the results stated in what follows are given in Section 4.1.

Denote $\ell=\mathbf{E}^{0}\left[L_{0}(n)\right]=\mathbf{E}^{0}\left[L_{0}(0)\right]$.

Proposition 3.1. Assume $\Phi$ to be a Poisson p.p., $F$ to be exponential and the noise $W$ to be bounded away from $0: \mathbf{P}\{W>w\}=1$ for some $w>0$. Let the path-loss function be given by (1). Then $\mathbf{P}^{0}\left\{L_{0}(0) \geq q\right\} \geq 1 / q$ for $q$ large enough.

Corollary 3.3. Under the assumptions of Proposition 3.1, we have:

- The mean exit delay from the typical node is infinite; $\ell=\infty$.

- In any given subset of plane with positive Lebesgue measure, at a given time, the expected number of points of $\Phi$ which have exit delays larger than $q$ decreases 
not faster than $1 / q$ asymptotically for large $q$.

The fact that the mean exit delay from the typical point is infinite $(\ell=\infty)$ seems to be a consequence of the potential existence of arbitrarily large "voids" (disks without points of $\Phi)$ around this point. Indeed, when conditioning on the existence of another point in the configuration $\Phi$, one obtains finite mean local delays. This will be shown in Proposition 3.2 below.

Before stating it we need to formalise the notion of existence of two given points $X, Y \in \mathbb{R}^{2}$ of $\Phi$. For this, we consider $\Phi$ under the two-fold Palm probability $\mathbf{P}^{X, Y}$. Since our results on the matter bear only on the Poisson p.p. case, we can assume (by Slivnyak's Theorem) the following version of the Palm probability of the Poisson p.p. $\Phi:$

$$
\mathbf{P}^{X, Y}\{\Phi \in \cdot\}=\mathbf{P}\{\Phi \cup\{X, Y\} \in \cdot\} .
$$

Moreover, under $\mathbf{P}^{X, Y}$, the marked Poisson p.p. $\tilde{\Phi}$ is obtained by an independent marking of the points of $\Phi \cup\{X, Y\}$ according to the original distribution of marks. Slightly abusing the notation, we denote by $L_{X, Y}(n)$ the local delay from $X$ to $Y$ at time $n$ when considered under $\mathbf{P}^{X, Y}$. Similar convention will be adopted in the notation of other types of delays under the Palm probabilities $\mathbf{P}^{X}$ or $\mathbf{P}^{X, Y}$.

Proposition 3.2. Assume $\Phi$ to be a Poisson p.p., $F$ to be exponential and the noise $W$ to have a general distribution. Then for all $X, Y \in \mathbb{R}^{2}$, the mean local delay from $X$ to $Y$ is finite given the existence of these two points in $\Phi$. More precisely,

$$
\mathbf{E}^{X, Y}\left[L_{X, Y}(0)\right]<\infty .
$$

The next result follows immediately from (6).

Corollary 3.4. Under the assumptions of Proposition 3.2,

$$
\mathbf{E}^{X, Y}\left[L_{X}(0)\right] \leq \mathbf{E}^{X, Y}\left[P_{X, Y}(0)\right]<\mathbf{E}^{X, Y}\left[L_{X, Y}(0)\right]<\infty .
$$

The following result is our main "negative" result concerning $\mathbb{G}$ in the Poisson p.p. case: 
Proposition 3.3. Under the assumptions of Proposition 3.1, we have

$$
\lim _{|X-Y| \rightarrow \infty} \frac{\mathbf{E}^{X, Y}\left[P_{X, Y}(0)\right]}{|X-Y|}=\infty
$$

In other words, the expected shortest delay necessary to send a packet between two given points of the Poisson p.p. grows faster than the Euclidean distance between these two points.

\subsection{Filling in Poisson Voids}

In this section we show that adding an independent periodic pattern of points to the Poisson p.p. allows one to get a linear scaling of the shortest path delay with Euclidean distance. In order to prove the existence and finiteness of the associated time constant, we adopt a slightly different approach to the notion of paths on $\mathbb{G}$, which will allow us to exploit a subadditive ergodic theorem. The proofs of the results stated in what follows are given in Section 4.2.

For $x \in \mathbb{R}^{2}$, let $X(x)$ be the point of $\Phi$ which is closest to $x$. The point $X(x) \in \Phi$ is a.s. well defined for all given $x \in \mathbb{R}^{2}$ since $\Phi$ is assumed simple and stationary p.p. For all $x, y \in \mathbb{R}^{2}$, define $P(x, y, n)=P_{X(x), X(y)}(n)$ to be the length of a shortest path of $\mathbb{G}$ from vertex $(X(s), n)$ to the set $\{(X(y), n+l), l \in \mathbb{N}\}$. We will call $P(x, y, n)$ the delay from $x$ to $y$ at time $n$. For all triples of points $x, y, z \in \mathbb{R}^{2}$, we have

$$
P(x, z, n) \leq P(x, y, n)+P(y, z, n+P(x, y, n)) .
$$

Let

$$
p(x, y, \Phi)=\mathbf{E}[P(x, y, 0) \mid \Phi] .
$$

Using the strong Markov property, we get that, conditionally on $\Phi$, the law of $P(y, z, n+$ $P(x, y, n))$ is the same as that of $P(y, z, n)$. Then, the last relation and (11) give

$$
p(x, z, \Phi) \leq p(x, y, \Phi)+p(y, z, \Phi) .
$$

We are now in a position to use the subadditive ergodic theorem to show the 
existence of the time constant

$$
\kappa_{\mathrm{d}}=\lim _{t \rightarrow \infty} \frac{p(0, t \mathrm{~d}, \Phi)}{t}
$$

where $\kappa_{\mathrm{d}}$ may depend on the unit vector $\mathrm{d} \in \mathbb{R}^{2}$ representing the direction in which the delay is measured. Here is the main result of this section.

Proposition 3.4. Consider the Poisson+Grid p.p. defined in Section 2.1 with exponential fading $F$ and with the path-loss function be given by (1). Then, for all unit vectors $\mathrm{d} \in \mathbb{R}^{2}$, the non-negative limit $\kappa_{\mathrm{d}}$ exists and is $\mathbf{P}$-a.s. finite. The convergence also holds in $L_{1}$.

Notice that $\kappa_{\mathrm{d}}$ is not a constant. Indeed, the superposition of the p.p.s $\Phi=\Phi_{M}$ and $\Phi_{G}$ is ergodic but not mixing due to the fact that $\Phi$ is a (stationary) grid. For d parallel to say the horizontal axis of the grid $\Phi_{G}$, the limit $\kappa_{\mathrm{d}}$ will depend on the distance from the line $\{t \mathrm{~d}: t \in \mathbb{R}\}$ to the nearest parallel (horizontal) line of the grid $\Phi_{G}$, i.e. on the shift $U_{G}$ of the grid. Here is a more precise formulation of the result.

Proposition 3.5. Under the assumptions of Proposition 3.4, the limit $\kappa_{\mathrm{d}}=\kappa_{\mathrm{d}}\left(U_{G}\right)$ is measurable w.r.t. the shift $U_{G}$ of the grid p.p. $\Phi_{G}$ and does not depend on the Poisson component $\Phi_{M}$ of the p.p. $\Phi$. Moreover, the set of vectors $\mathrm{d}$ in the unit sphere for which $\kappa_{\mathrm{d}}\left(U_{G}\right)$ is not $\mathbf{P}$-a.s. a constant is at most countable.

The last result on this case is:

Proposition 3.6. Under the assumptions of Proposition 3.4, suppose that $W$ is constant and strictly positive. Then $\mathbf{E}\left[\kappa_{\mathrm{d}}\right]>0$.

Finally let us remark that the method used in this section cannot be used in the case of the Poisson p.p. (without the addition of the grid point process). The main problem is the lack of integrability of $p(x, y, \Phi)$ as stated in the following result. Note however, that this does not imply immediately that $\kappa_{\mathrm{d}}=\infty$.

Corollary 3.5. Under the assumptions of Proposition $3.1 \mathbf{E}[p(x, y, \Phi)]=\infty$ for all $x$ and $y$ in $\mathbb{R}^{2}$. 


\section{Proofs}

Consider the shortest path from $\left(X_{i}, n\right)$ to $\left(\Phi \backslash\left\{X_{i}\right\}\right) \times \mathbb{Z}$. Let $\mathcal{T}_{i}(n)$ be the number of edges $\left(X_{i}, k\right),\left(X_{i}, k+1\right)$ in this path such that $e_{i}(k)=1$. These variables are the number of trials before the first exit form $X_{i}$ at time $n$. Obviously

$$
\mathcal{T}_{i}(n) \leq L_{i}(n)
$$

We will also consider an auxiliary graph $\widehat{\mathbb{G}}$, called the (space-time) Signal to Noise Ratio (SNR) graph, defined exactly in the same manner as the SINR graph $\mathbb{G}$ except that the variables $\operatorname{SINR}_{i, j}(n)$ defined in (15) are replaced by the variables

$$
\operatorname{SNR}_{i, j}(n)=\frac{F_{i, j}(n) / l\left(\left|X_{i}-X_{j}\right|\right)}{W_{j}(n)}
$$

Note that this modification consists in suppressing the interference term $I_{i, j}(n)$ in the SINR condition in (4). The edges of $\mathbb{G}$ form a subset of the edges of $\widehat{\mathbb{G}}$ (both graph share the same vertexes), which will be denoted by

$$
\mathbb{G} \subset \widehat{\mathbb{G}}
$$

In what follows we will denote the delays, local delays, exit delays and numbers of trials

related to $\widehat{\mathbb{G}}$ by $\widehat{P}_{i, j}(n), \widehat{L}_{i, j}(n), \widehat{L}_{i}(n)$ and $\widehat{\mathcal{T}}_{i}(n)$, respectively. The inclusion $\mathbb{G} \subset \widehat{\mathbb{G}}$ implies immediately that $\widehat{P}_{i, j}(n) \leq P_{i, j}(n)$ and the same inequalities hold for the three other families of variables mentioned above.

\subsection{Proofs of Results of Section 3.2}

Proof. (of Proposition 3.1) The inclusion (16) and the inequality (14) yield

$$
\widehat{\mathcal{T}}_{i}(n) \leq \mathcal{T}_{i}(n) \leq L_{i}(n),
$$

which holds for all $i, n$. The results follow from the above inequalities and the next lemma.

Lemma 4. Under the assumptions of Proposition 3.1, $\mathbf{P}^{0}\left\{\widehat{\mathcal{T}}_{0}(0) \geq q\right\} \geq 1 / q$ for $q$ 
large enough.

Proof. Under $\mathbf{P}^{0}$, denote by $\tau_{k}$ the $k$ th time slot in $\{0,1, \ldots\}$, such that $e_{0}(k)=1$. For all $q \geq 0$ we have

$$
\begin{aligned}
\mathbf{P}^{0}\left\{\widehat{\mathcal{T}}_{0}(0)>q \mid \Phi\right\} & =\mathbf{P}^{0}\left\{\forall_{0 \leq k \leq q} \forall_{0 \neq X_{i} \in \Phi} \delta_{0, i}\left(\tau_{k}\right)=0 \mid \Phi\right\} \\
& =\mathbf{P}^{0}\left\{\forall_{0 \leq k \leq q} \forall_{0 \neq X_{i} \in \Phi} e_{i}\left(\tau_{k}\right)=1 \text { or } \operatorname{SNR}_{0, i}\left(\tau_{k}\right)<T \mid \Phi\right\}
\end{aligned}
$$

and by the conditional independence of marks given $\Phi$

$$
\begin{aligned}
\mathbf{P}^{0}\left\{\widehat{\mathcal{T}}_{0}(0)>q \mid \Phi\right\} & =\prod_{0 \neq X_{i} \in \Phi}\left(p+(1-p) \mathbf{P}\left\{F<T l\left(\left|X_{i}\right|\right) W\right\}\right)^{q} \\
& =\exp \left\{q \sum_{0 \neq X_{i} \in \Phi} \log \left(p+(1-p)\left(1-e^{-\mu T l\left(\left|X_{i}\right|\right) W}\right)\right)\right\},
\end{aligned}
$$

where $F, W$ are independent generic random variables representing fading and thermal noise, independent of $\Phi, F$ is exponential with mean $1 / \mu$, Using the Laplace functional formula for $\Phi$ and the assumption that $W>w$ a.s. we have

$$
\begin{aligned}
\mathbf{P}^{0}\left\{\widehat{\mathcal{T}}_{0}(0) \geq q\right\} & \geq \exp \left(-2 \pi \lambda \int_{v>0}\left(1-\left(1-(1-p) e^{-w \mu l(v) T}\right)^{q}\right) v \mathrm{~d} v\right) \\
& =\exp \left(-\pi \lambda \int_{v>0}\left(1-(1-f(v))^{q}\right) \mathrm{d} v\right)
\end{aligned}
$$

where

$$
f(v):=(1-p) \exp \left(-K v^{\beta / 2}\right) \text { and } \quad K=w \mu T A^{\beta} .
$$

In what follows we will show that the expression in (17) is not smaller than $1 / q$ for $q$ large enough. To this regard denote by $v_{q}$ the unique solution of $f(v)=\frac{1}{q}$. We have

$$
v_{q}=\frac{1}{A^{2}(\mu T w)^{2 / \beta}}(\log (q(1-p)))^{2 / \beta} .
$$

It is clear that $f(v)$ tends to 0 when $v$ tends to infinity and that $v_{q}$ tends to infinity as $q$ tends to infinity. Therefore, there exists a constant $Q=Q(\mu, w, A, T)<\infty$ such 
that for all $q \geq Q$ and for all $v \geq v_{q}$,

$$
(1-f(v)) \geq \exp (-f(v))
$$

Hence, for all $q \geq Q$,

$$
\begin{aligned}
\int_{v>0}\left(1-(1-f(v))^{q}\right) \mathrm{d} v & \leq v_{q}+\int_{v_{q}}^{\infty}\left(1-(1-f(v))^{q}\right) \mathrm{d} v \\
& \leq v_{q}+\int_{v=v_{q}}^{\infty}(1-\exp (-q f(v)) \mathrm{d} v \\
& \leq v_{q}+\int_{v_{q}}^{\infty} q f(v) \mathrm{d} v \\
& =v_{q}+\int_{u=0}^{\infty} q f\left(u+v_{q}\right) \mathrm{d} u
\end{aligned}
$$

The third inequality follows from the fact that $1-\exp (-x) \leq x$. Using now the fact that $\left(u+v_{q}\right)^{\beta / 2} \geq u+v_{q}^{\beta / 2}$ (for $q$ large enough, say again $q \geq Q$ ) we get that

$$
\begin{aligned}
\int_{u=0}^{\infty} q f\left(u+v_{q}\right) \mathrm{d} u & \left.=\int_{u=0}^{\infty} q(1-p) \exp \left(-K\left(u+v_{q}\right)^{\beta / 2}\right)\right) \mathrm{d} u \\
& \leq \int_{u=0}^{\infty} q(1-p) \exp \left(-K u-K v_{q}^{\beta / 2}\right) \mathrm{d} u=\frac{1}{K}
\end{aligned}
$$

since $(1-p) \exp \left(-K v_{q}^{\beta / 2}\right)=1 / q$. Hence for $q \geq Q$

$$
\int_{v>0}\left(1-(1-f(v))^{q}\right) \mathrm{d} v \leq v_{q}+\frac{\alpha}{K}
$$

Also it is not difficult to see that $\beta>2$ implies

$$
v_{q} \leq \frac{\log q}{\pi \lambda}-\frac{1}{K}
$$

for $q$ large enough. This implies for $q$ large enough, say again $q \geq Q$,

$$
\exp \left(-\pi \lambda \int_{v>0}\left(1-(1-f(v))^{q}\right) \mathrm{d} v\right) \geq \exp \left(-\pi \lambda\left(v_{q}+1 / K\right)\right) \geq \frac{1}{q}
$$

which completes the proof.

Proof. (of Proposition 3.2). Assume without loss of generality $Y=0$ and $|X|=r$. 
Under $\mathbf{P}$, consider the p.p. $\Phi \cup\{X, 0\}$ and its independent marking. Given $\Phi$, the r.v. $L_{X, 0}(0)$ associated with the independently marked p.p. $\Phi \cup\{X, 0\}$ has a geometric distribution with parameter

$$
\left.\pi_{X, 0}(\Phi)=p(1-p) \operatorname{Pr}\{F \geq l(r)(W+I))\right\}
$$

where $F, W, I$ are independent r.v.s, $F, W$ are generic fading and noise variables and $I=\sum_{X_{i} \in \Phi} e_{i}(0) F_{i, 0}(0) / l\left(\left|X_{i}\right|\right)$. Using the exponential distribution of $F$ and the independence, we obtain

$$
\pi_{X, 0}(\Phi)=\mathbf{E}\left[e^{-\mu l(r) T W}\right] \mathbf{E}\left[e^{-\mu l(r) T I} \mid \Phi\right]
$$

The mean of the geometric r.v. is known to be $\mathbf{E}^{X, 0}\left[L_{X, 0}(0) \mid \Phi\right]=1 / \pi_{X, 0}(\Phi)$. By unconditioning with respect to $\Phi$, one obtains

$$
\mathbf{E}^{X, 0}\left[L_{X, 0}(0)\right]=\frac{1}{\mathcal{L}_{W}(\mu l(r) T)} \mathbf{E}\left[\frac{1}{\mathbf{E}\left[e^{-\mu l(r) T I} \mid \Phi\right]}\right] .
$$

The first factor in the above expression is obviously finite. In what follows we will evaluate the second one.

By the conditional independence of marks and denoting by $\mathcal{L}_{e F}(\cdot)$ is the Laplace transform of $e F$, where $e, F$ are independent generic variables for $e_{i}(0)$ and $F_{i, 0}(0)$ we have

$$
\begin{aligned}
\left.\left(\mathbf{E}\left[e^{-\mu l(r) T I} \mid \Phi\right]\right]\right)^{-1} & =\left(\mathbf{E}\left[\exp \left(-\mu l(r) T \sum_{X_{i} \in \Phi} e_{i}(0) F_{i, 0}(n) / l\left(\left|X_{i}\right|\right)\right) \mid \Phi\right]\right)^{-1} \\
& =\exp \left(\sum_{X_{i} \in \Phi} \log \mathcal{L}_{e F}\left(\mu T l(r) / l\left(\left|X_{i}\right|\right)\right)\right)
\end{aligned}
$$

Note that $\mathcal{L}_{e F}(\xi)=1-p+p \mathcal{L}_{F}(\xi)=1-p+p \mu /(\mu+\xi)$. Using this and the Laplace functional formula for $\Phi$, (cf. [9, Eq. 9.4.17]) we obtain

$$
\mathbf{E}\left[\frac{1}{\mathbf{E}\left[e^{-\mu l(r) T I} \mid \Phi\right]}\right]=\exp \left\{2 \pi p \lambda \int_{0}^{\infty} \frac{v T l(r)}{l(v)+(1-p) T l(r)} \mathrm{d} v\right\}
$$

(cf. (2) Using now the fact that for the Poisson p.p., $\breve{M}_{[2]}(\mathrm{d} x)=\lambda \mathrm{d} x$ ), it is now easy 
to see that for any path-loss function satisfying $\int_{\epsilon}^{\infty} v / l(v) \mathrm{d} v<\infty$, the integral in the exponent of the last expression is finite. This completes the proof.

Proof. (of Proposition 3.3). Using the the inclusion (16), inequality (14) and the left-hand side of (6) and we have

$$
\widehat{\mathcal{T}}_{i}(n) \leq \mathcal{T}_{i}(n) \leq L_{i}(n) \leq P_{i, j}(n)
$$

Thus, it is enough to show

$$
\lim _{|X-Y| \rightarrow \infty} \frac{\mathbf{E}^{X, Y}\left[\widehat{\mathcal{T}}_{X}(0)\right]}{|X-Y|}=\infty
$$

Without loss of generality assume $X=0$ and $|Y|=r$. Using the same arguments as in the proof of Lemma 4 and the representation (7) of the Palm probability with respect to Poisson p.p., we obtain

$$
\begin{aligned}
\mathbf{P}^{0, Y} & \left\{\widehat{\mathcal{T}}_{0}(0)>q \mid \Phi\right\} \\
& \geq \prod_{0, Y \neq X_{i} \in \Phi}\left(p+(1-p) \mathbf{P}\left\{F<T l\left(\left|X_{i}\right|\right) W\right\}\right)^{q}(p+(1-p) \mathbf{P}\{F<T l(|Y|) W\})^{q} \\
& \geq \exp \left(-\pi \lambda \int_{v>0}\left(1-(1-f(v))^{q}\right) \mathrm{d} v\right) \alpha(r)^{q},
\end{aligned}
$$

where $\alpha(r)=1-(1-p) e^{-w \mu A^{\alpha} T r^{\beta}}$. Using (19), which holds for large $q$, more precisely $q>Q=Q(\mu, w, A, T)$, we obtain

$$
\frac{\mathbf{E}^{0, Y}\left[\widehat{\mathcal{T}}_{0}(0)\right]}{r} \geq \frac{1}{r} \sum_{q>Q} \frac{\alpha(r)^{q}}{q} .
$$

It is now easy to see that

$$
\lim _{r \rightarrow \infty} \frac{1}{r} \sum_{q>Q} \frac{\alpha(r)^{q}}{q}=\infty
$$

\subsection{Proofs of Results of Section 3.3}

Denote by $B_{x}(R)$ the ball centered at $x \in \mathbb{R}^{2}$ of radius $R$. Similarly as for the delays, we extend the definition of the local delays to arbitrary pairs of points $x, y \in \mathbb{R}^{2}$ by 
taking $L(x, y, n)=L_{X(x), X(y)}(n)$. We first establish the following technical result:

Lemma 5. Under the assumptions of Proposition 3.4 let $X_{i}, X_{j} \in \Phi \cap B_{0}(R)$ for some $R>0$, where $\Phi=\Phi_{M}+\Phi_{G_{s}}$. Then the conditional expectation of the local delay $L_{i, j}(0)$ given $\Phi$ satisfies

$$
\begin{aligned}
\mathbf{E}\left[L_{i, j}(0) \mid \Phi\right] & \frac{1}{p(1-p) \mathcal{L}_{W}\left(T \mu A^{\beta}\left|X_{i}-X_{j}\right|^{\beta}\right)} \exp \left\{-\sum_{\Phi \ni X_{k}, k \neq i, k} \log \mathcal{L}_{e F^{\prime}}\left(\frac{T\left|X_{i}-X_{j}\right|^{\beta}}{\left|X_{j}-X_{k}\right|^{\beta}}\right)\right\} \\
\leq & \frac{1}{p(1-p) \mathcal{L}_{W}\left(T \mu(A 2 R)^{\beta}\right)} \\
& \times e^{-49 \log (1-p)+(2 R)^{\beta} p T C(s, \beta)} \\
& \times e^{-\Phi_{M}\left(B_{0}(2 R)\right) \log (1-p)} \\
& \times \exp \left\{-\sum_{X_{k} \in \Phi_{M},\left|X_{k}\right|>2 R} \log \left(1-p+\frac{p\left(\left|X_{k}\right|-R\right)^{\beta}}{\left(\left|X_{k}\right|-R\right)^{\beta}+T(2 R)^{\beta}}\right)\right\}
\end{aligned}
$$

where $C(s, \beta)<\infty$ is some constant (which depends on $s$ and $\beta$ but not on $\Phi$ ), $F^{\prime}$ is an exponential random variable of mean 1 and $\mathcal{L}_{e F^{\prime}}(\cdot)$ is the Laplace transform of $e F^{\prime}$.

Proof. We first prove the equality in (20). When using the independence assumptions, we have

$$
\begin{aligned}
& \mathbf{P}\left\{L_{i, j}(0)>m \mid \Phi\right\} \\
&= \mathbf{P}\left\{\forall _ { n = 1 } ^ { m } \left(e_{j}(n)=1\right.\right. \text { or } \\
&\left.\left.e_{j}(n)=0 \text { and } e_{i}(n) F_{i, j}(n) \leq T l\left(\left|X_{i}-X_{j}\right|\right)\left(W_{j}(n)+I_{i, j}(n)\right)\right)\right\} \\
&=\prod_{n=1}^{m}\left(p+(1-p)\left(1-p+p\left(1-\mathcal{L}_{W}\left(T \mu A^{\beta}|x-y|^{\beta}\right)\right.\right.\right. \\
&\left.\left.\left.\times \prod_{\Phi \ni X_{k}, k \neq i, j} \mathcal{L}_{e F^{\prime}}\left(\frac{T\left|X_{i}-X_{j}\right|^{\beta}}{\left|X_{j}-X_{k}\right|^{\beta}}\right)\right)\right)\right) .
\end{aligned}
$$

The result then follows from the evaluation of

$$
\mathbf{E}\left[L_{i, j}(0) \mid \Phi\right]=\sum_{m=0}^{\infty} \mathbf{P}\left\{L_{i, j}(0)>m \mid \Phi\right]
$$

The bound $\left|X_{i}-X_{j}\right| \leq 2 R$ used in the Laplace transform of $W$ leads to the first 
factor of the upper bound. We now factorize the exponential function in (20) as the product of three exponential functions

$$
\begin{aligned}
\alpha & :=\exp \left\{-\sum_{\Phi_{G_{s}} \ni X_{k}, k \neq i, j}\right\}, \\
\beta & :=\exp \left\{-\sum_{\Phi_{M} \ni X_{k}, k \neq i, j\left|X_{k}\right| \leq 2 R}\right\}, \\
\gamma & :=\exp \left\{-\sum_{\Phi_{M} \ni X_{k},\left|X_{k}\right|>2 R}\right\} .
\end{aligned}
$$

Next we prove that the last three exponentials are upper-bounded by (a), (b) and (c) in (20), respectively.

(a) We use $\left|X_{i}-X_{j}\right| \leq 2 R$ and Jensen's inequality to get

$$
\begin{aligned}
\log \mathcal{L}_{e F^{\prime}}\left(\frac{T\left|X_{i}-X_{j}\right|^{\beta}}{\left|X_{j}-X_{k}\right|^{\beta}}\right) & \geq \log \mathcal{L}_{e F^{\prime}}\left(\frac{T(2 R)^{\beta}}{\left|X_{j}-X_{k}\right|^{\beta}}\right) \\
& \geq \frac{-T(2 R)^{\beta} \mathbf{E}\left[e F^{\prime}\right]}{\left|X_{j}-X_{k}\right|^{\beta}} \\
& =-p T(2 R)^{\beta}\left|X_{j}-X_{k}\right|^{-\beta}
\end{aligned}
$$

We now prove that

$$
\sum_{\Phi_{G_{s}} \ni X_{k}:\left|X_{j}-X_{k}\right|>3 \sqrt{2} s}\left|X_{j}-X_{k}\right|^{-\beta} \leq C(s, \beta),
$$

for some constant $C(s, \beta)$. This follows from an upper-bounding of the value of $\left|X_{j}-X_{k}\right|^{-\beta}$ by the value of the integral $1 / s^{2} \int\left(\left|X_{j}-x\right|-\sqrt{2} s\right)^{-\beta} \mathrm{d} x$ over the square with corner points $X_{k}, X_{k}+(s, 0), X_{k}+(0, s)$ and $X_{k}+(s, s)$. In this way we obtain

$$
\begin{aligned}
& \sum_{\Phi_{G_{s} \ni X_{k}:\left|X_{j}-X_{k}\right|>3 \sqrt{2} s}\left|X_{j}-X_{k}\right|^{-\beta}} \leq \frac{1}{s^{2}} \int_{\left|x-X_{j}\right|>2 \sqrt{2} s}^{\infty}\left(\left|X_{j}-x\right|-\sqrt{2} s\right)^{-\beta} \mathrm{d} x \\
&=\frac{2 \pi}{s^{2}} \int_{\sqrt{2} s}^{\infty} \frac{t+\sqrt{2} s}{t^{\beta}} \mathrm{d} t=: C(s, \beta)<\infty .
\end{aligned}
$$


Combining this and what precedes, we get that

$$
\exp \left\{-\sum_{X_{k} \in \Phi_{G_{s}},\left|X_{j}-X_{k}\right|>2 \sqrt{s}} \log \mathcal{L}_{e F^{\prime}}\left(\frac{T\left|X_{j}-X_{i}\right|^{\beta}}{\left|X_{j}-X_{k}\right|^{\beta}}\right)\right\} \leq \exp \left(T(2 R)^{\beta} C(s, \beta)\right)
$$

We also have

$$
\log \mathcal{L}_{e F^{\prime}}\left(\frac{T(2 R)^{\beta}}{\left|y-X_{i}\right|^{\beta}}\right) \geq \log \mathcal{L}_{e F^{\prime}}(\infty)=\log (1-p)
$$

for all $X_{k} \in \Phi_{G_{s}}$ and in particular for $\left|X_{j}-X_{k}\right| \leq 3 \sqrt{2} s$. Hence we obtain

$$
\exp \left\{-\sum_{X_{k} \in \Phi_{G_{s}}}(\ldots)\right\} \leq e^{-49 \log (1-p)+T(2 R)^{\beta} C(s, \beta)},
$$

where 49 upper-bounds the number of points $X_{k} \in \Phi_{G_{s}}$ such that $\left|X_{j}-X_{k}\right| \leq$ $3 \sqrt{2} s$.

(b) Using the bound $\left|X_{j}-X_{i}\right| \leq 2 R$ and the inequality $\log \mathcal{L}_{e F^{\prime}}(\xi) \geq \log \mathcal{L}_{e F^{\prime}}(\infty)=$ $\log (1-p)$, we obtain

$$
\exp \left\{-\sum_{\Phi_{M} \ni X_{k}, k \neq i, j,\left|X_{i}\right| \leq 2 R}(\ldots)\right\} \leq e^{-\Phi_{M}\left(B_{0}(2 R)\right) \log (1-p)} .
$$

(c) Using the bounds $\left|X_{j}-X_{i}\right| \leq 2 R$ and $\left|X_{j}-X_{k}\right| \geq\left|X_{k}\right|-R$ (the latter follows from the triangle inequality) and the expression $\mathcal{L}_{e F^{\prime}}(\xi)=1-p+\frac{p}{1+\xi}$, we obtain

$$
\begin{aligned}
& \exp \left\{-\sum_{\Phi_{M} \ni X_{k},\left|X_{k}\right|>2 R}(\ldots)\right\} \\
& \leq \exp \left\{-\sum_{X_{k} \in \Phi_{M},\left|X_{k}\right|>2 R} \log \left(1-p+\frac{p\left(\left|X_{k}\right|-R\right)^{\beta}}{\left(\left|X_{k}\right|-R\right)^{\beta}+T(2 R)^{\beta}}\right)\right\} .
\end{aligned}
$$

This completes the proof.

We can now prove the following auxiliary result. 
Lemma 6. Under the assumptions of Proposition 3.4 for all points $x, y$ of $\mathbb{R}^{2}$,

$$
\mathbf{E}\left[\sup _{x_{1}, y_{1} \in[x, y]} p\left(x_{1}, y_{1}, \Phi\right)\right]<\infty,
$$

where the supremum is taken over $x_{1}, y_{1}$ belonging to the interval $[x, y] \subset \mathbb{R}^{2}$.

Proof. Without loss of generality, we assume that $(x+y) / 2=0$ is the origin of the plane. Let $B=B_{0}(R)$ be the ball centered at 0 and of radius $R$ such that no modification of the points in the complement of $B$ modifies $X(z)$ for any $z \in[x, y]$ (recall that $X(z)$ is the point of $\Phi$ which is the closest from $z$ ). Since $\Phi=\Phi_{M}+\Phi_{G_{s}}$, with $\Phi_{G_{S}}$ the square lattice p.p. with intensity $1 / s^{2}$, it suffices to take $R=|u-v| / 2+$ $\sqrt{2} s$. Let $B^{\prime}=B_{0}(2 R)$. By the above choice of $B$ and the inequality (6) we have for all $x_{1}, y_{1} \in[x, y]$

$$
P\left(x_{1}, y_{1}, 0\right) \leq \sum_{X_{i}, X_{j} \in \Phi \cap B} L_{i, j}(0)
$$

and consequently

$$
\sup _{x_{1}, y_{1} \in[x, y]} p\left(x_{1}, y_{1}, \Phi\right) \leq \sum_{X_{i}, X_{j} \in \Phi \cap B} \mathbf{E}\left[L_{i, j}(0) \mid \Phi\right]
$$

Using the result of Lemma 20 we obtain

$$
\begin{aligned}
& \sup _{x_{1}, y_{1} \in[x, y]} \overline{\left|p^{*}\left(x_{1}, y_{1}, \Phi\right)\right|} \\
& \leq \frac{e^{-49 \log (1-p)+(2 R)^{\beta} p T C(s, \beta)}}{p(1-p) \mathcal{L}_{W}\left(T \mu A(2 R)^{\beta}\right)} \\
& \quad \times \exp \left\{-\sum_{X_{k} \in \Phi_{M},\left|X_{k}\right|>2 R} \log \left(1-p+\frac{p\left(\left|X_{k}\right|-R\right)^{\beta}}{\left(\left|X_{k}\right|-R\right)^{\beta}+T(2 R)^{\beta}}\right)\right\} \\
& \quad \times\left(\Phi_{M}(B)+\pi(R+\sqrt{2} s)^{2} / s^{2}\right) e^{-\Phi_{M}\left(B^{\prime}\right) \log (1-p)},
\end{aligned}
$$

where $\pi(R+\sqrt{2} s)^{2} / s^{2}$ is an upper bound of the number of points of $\Phi_{G_{s}}$ in $B$. The first factor in the above upper bound is deterministic. The two other factors are random and independent due to the independence property of the Poisson p.p. The finiteness of the expectation of the last expression follows from the finiteness of the exponential moments (of any order) of the Poisson random variable $\Phi_{M}\left(B^{\prime}\right)$. For the expectation 
of the second (exponential) factor, we use the known form of the Laplace transform of the Poisson SN to obtain the following expression

$$
\mathbf{E}\left[\exp \left\{-\sum(\ldots)\right\}\right]=\exp \left\{2 \pi p \lambda_{M} \int_{R}^{\infty} \frac{T(2 R)^{\beta}}{v^{\beta}+(1-p) T(2 R)^{\beta}}(v+R) \mathrm{d} v\right\}<\infty .
$$

Proof. (of Proposition 3.4 The existence and finiteness of the limit $\kappa_{\mathrm{d}}$ follows from the subadditivity (13) and Lemma 6 by the continuous-parameter sub-additive ergodic theorem (see [19, Theorem 4]).

Proof. (of Proposition 3.5) First, we prove the second statement; i.e., that $\kappa_{\mathrm{d}}$ is constant for all $d$ in the unit sphere off some countable subset. Note that the point process $\Phi$ is ergodic as the independent superposition of mixing Poisson p.p. $\Phi_{M}$ and ergodic grid process $\Phi_{G}$. This can be easily proved using e.g. the respective characterisations of above properties by means of Laplace transforms of p.p. (see [9, Prop. 12.3.VI]). From the ergodicity of $\Phi$ we cannot conclude the desired property for any vector $\mathrm{d}$ since the limit $\kappa_{\mathrm{d}}=\kappa_{\mathrm{d}(\Phi)}$ is not necessarily invariant with respect to translations of $\Phi$ by any vector $x \in \mathbb{R}^{2}$ but only $x=\alpha \mathrm{d}$ for any scalar $\alpha \in \mathbb{R}$. The announced result follows from [22, Th. 1].

For the first statement, consider a product space with on which two independent p.p.s $\left(\Phi_{M}, \Phi_{G}\right)$ are defined. Fix some vector $\mathrm{d}$ and define the operator $T=T_{1} \times T_{2}$ on this product space as the product of two operators, which correspond to the shift in the direction d, of $\Phi_{M}$ and $\Phi_{G}$ respectively. The $\sigma$-field invariant with respect to $T$ is the product of the respective $\sigma$-fields invariant with respect to $T_{1}$ and $T_{2}$. The latter is trivial since $\Phi_{M}$ is mixing (as a Poisson p.p.). Consequently every function of $\left(\Phi_{M}, \Phi_{G}\right)$ that is invariant with respect to the shift in the direction $\mathrm{d}$ of its first argument $\left(\Phi_{M}\right)$ is a.s. constant. This concludes the proof that $\kappa_{\mathrm{d}}$ is constant in $\Phi_{M}$ and thus depends only on $U_{G}$.

Proof. (of Proposition 3.6) For a given path $\sigma=\left\{\left(X_{0}, n_{0}\right),\left(X_{1}, n_{0}+1\right), \ldots,\left(X_{k}, n_{0}+\right.\right.$ $k)\}$ on $\mathbb{G}$ denote by $|\sigma|=\sum_{i=1}^{k}\left|X_{i}-X_{i-1}\right|$ the Euclidean length of the projection of $\sigma$ on $\mathbb{R}^{2}$; let us call it Euclidean length of $\sigma$ for short and recall that the (graph) length of $\sigma$ is equal to $k$. For fixed $\epsilon>0$ and all $n \geq 1$ denote by $\Pi(n)=\Pi_{\epsilon}(n)$ the event that 
there exists a path on $\mathbb{G}$ starting at $(X(0), 0)$ that has (graph) length $n$ and Euclidean length larger than $n / \epsilon$.

Assume $\mathbf{E}\left[\kappa_{\mathrm{d}}\right]=0$. We show first that this implies that for any $\epsilon>0, \mathbf{P}^{0}$-a.s. the event $\Pi_{\epsilon}(n)$ holds for infinitely many $n$

$$
\mathbf{P}^{0}\left\{\bigcap_{n \geq 1} \bigcup_{k \geq n} \Pi_{\epsilon}(k)\right\}=1
$$

Indeed, $\mathbf{E}\left[\kappa_{\mathrm{d}}\right]=0$ implies $\kappa_{\mathrm{d}}=0 \mathbf{P}$-a.s. and by Palm-Matthes definition of the Palm probability $\mathbf{P}^{0}$-a.s. as well. This means that $\mathbf{E}^{0}[P(0, t \mathrm{~d}, 0) \mid \Phi] / t \rightarrow 0$, when $t \rightarrow \infty$, which implies that

$$
\lim _{k} P\left(0, t_{k} \mathrm{~d}, 0\right) / t_{k} \rightarrow 0
$$

$\mathbf{P}^{0}$-a.s. for some subsequence $\left\{t_{k}: k \geq 1\right\}$, with $\lim _{k} t_{k}=\infty$. Recall that $P\left(0, t_{k} \mathrm{~d}, 0\right)$ is the length of a shortest path from $(X(0), 0)$ (with $X(0)=0$ under $\left.\mathbf{P}^{0}\right)$ to $\left\{\left(X\left(t_{k} \mathrm{~d}\right), n\right)\right.$ : $n \geq 0\}$. Denote one of such shortest paths by $\sigma_{k}$. By the triangle inequality its Euclidean length satisfies

$$
\left|\sigma_{k}\right| \geq\left|0-X\left(t_{k} \mathrm{~d}\right)\right| \geq t_{k}-\sqrt{2} s
$$

From (22) and (23) one concludes that for any $\epsilon>0$ and $k$ large enough the length of the path $\sigma_{k}$ is smaller than $\epsilon$ time its Euclidean length $\left|\sigma_{k}\right|$. Now, (21) follows from the fact that the length of the path $\sigma_{k}$ tends to infinity with $k$, which is a consequence of $t_{k} \rightarrow \infty$ and the local finiteness of the graph $\mathbb{G}$ (cf Corollary 3.1).

We conclude the proof by showing that for $\epsilon$ small enough,

$$
\sum_{n} \mathbf{P}^{0}\left\{\Pi_{\epsilon}(n)\right\}<\infty
$$

which by the Borel-Cantelli lemma implies that $\Pi(n)$ holds $\mathbf{P}^{0}$-a.s. only for a finite number of integers $n$ and thus contradicts to (21). To this regard assume constant $W=w>0$ and let $\mathcal{P}_{w}^{n}$ denote the set of paths $\sigma$ of $\mathbb{G}$ of length $n$, originating from $(X(0)=0,0)$. Denote also by $\mathcal{P}_{0}^{n}$ the analogous set of paths on the graph constructed 
under assumption $W=0$. Note that by monotonicity,

$$
\mathcal{P}_{w}^{n} \subset \mathcal{P}_{0}^{n}
$$

By the definition

$$
\mathbf{P}^{0}\left\{\Pi_{\epsilon}(n) \mid \Phi\right\}=\mathbf{P}^{0}\left(\bigcup_{\sigma}\left\{\sigma \in \mathcal{P}_{w}^{n} \text { and }|\sigma| \geq n / \epsilon\right\} \mid \Phi\right)
$$

where the sum bears on all possible $n$-tuples $\sigma=\left(\left(X_{j_{1}}, 1\right), \ldots,\left(X_{j_{n}}, n\right)\right)$, with $X_{j_{i}} \in \Phi$. From this we have

$$
\begin{aligned}
\mathbf{P}^{0} & \left(\Pi_{\epsilon}(n) \mid \Phi\right) \\
& \leq \sum_{\sigma} \mathbf{P}^{0}\left\{\sigma \in \mathcal{P}_{W}^{n},|\sigma| \geq n / \epsilon \mid \Phi\right\} \\
& =\sum_{\sigma} \mathbf{P}^{0}\left\{\sigma \in \mathcal{P}_{W}^{n},|\sigma| \geq n / \epsilon \mid \Phi, \sigma \in \mathcal{P}_{0}^{n}\right\} \mathbf{P}^{0}\left\{\sigma \in \mathcal{P}_{0}^{n} \mid \Phi\right\} \\
& \leq \mathbf{E}^{0}\left[\mathcal{H}_{0}^{\text {out }, n ; W=0}(0) \mid \Phi\right] \sup _{\sigma} \mathbf{P}^{0}\left\{\sigma \in \mathcal{P}_{W}^{n},|\sigma| \geq n / \epsilon \mid \Phi, \sigma \in \mathcal{P}_{0}^{n}\right\},
\end{aligned}
$$

where $\mathcal{H}_{0}^{\text {out }, n ; W=0}(0)$ denotes the number of paths of length $n$ originating from $\left(X_{0}=\right.$ $0,0)$ under the assumption $W=0$. But

$$
\begin{aligned}
& \sup _{\sigma} \mathbf{P}^{0}\left\{\sigma \in \mathcal{P}_{w}^{n},|\sigma| \geq n / \epsilon \mid \Phi, \sigma \in \mathcal{P}_{0}^{n}\right\} \\
& \leq \sup _{\substack{\sigma=\left(\left(X_{j_{1}}, 1\right), \ldots,\left(X_{j_{n}}, n\right)\right) \\
\sum_{i=1}^{n}\left|X_{j_{i}}-X_{j_{i-1}}\right| \geq n / \epsilon}} \mathbf{E}^{0}\left[\prod_{i=1}^{n} \delta_{j_{i-1}, j_{i}}(i-1, w) \mid \Phi, \sigma \in \mathcal{P}_{0}^{n}\right],
\end{aligned}
$$

where $X_{j_{0}}=0$ and $\delta_{j_{i-1}, j_{i}}(i-1, w)=\delta_{j_{i-1}, j_{i}}(i-1)$ is the indicator of the existence of the edge from $\left(X_{j_{i-1}}, i-1\right)$ to $\left(X_{j_{i}}, i\right)$ defined by $(4)$, and where we add in the notation the dependence on the noise $W=w$. Using the conditional independence of marks, (4), (15) and lack of memory of the exponential distribution of $F$ of parameter $\mu$ we 
have for the path-loss function (1)

$$
\begin{aligned}
\mathbf{E}^{0}\left[\prod_{i=1}^{n} \delta_{j_{i-1}, j_{i}}(i-1, w) \mid \Phi, \sigma \in \mathcal{P}_{0}^{n}\right] & =\prod_{i=1}^{n} \mathbf{E}^{0}\left[\delta_{j_{i-1}, j_{i}}(i-1, w) \mid \Phi, \delta_{j_{i-1}, j_{i}}(i-1,0)=1\right] \\
& =\prod_{i=1}^{n} \exp \left(-\mu\left(A\left|X_{j_{i-1}}-X_{j_{i}}\right|\right)^{\beta} T w\right) .
\end{aligned}
$$

Hence

$$
\sup _{\sigma} \mathbf{P}^{0}\left\{\sigma \in \mathcal{P}_{w}^{n},|\sigma| \geq n / \epsilon \mid \Phi, \sigma \in \mathcal{P}_{0}^{n}\right\} \leq \exp \left(-\mu A^{\beta} T w n \epsilon^{-\beta}\right),
$$

where the last inequality follows from a convexity argument. Using this and (27), we get

$$
\begin{aligned}
\mathbf{E}^{0}\left(\Pi_{\epsilon}(n)\right) & \leq \mathbf{E}^{0}\left[\mathcal{H}_{0}^{\text {out }, n ; W=0}(0)\right] \exp \left(-\mu A^{\beta} T w n \epsilon^{-\beta}\right) \\
& \leq \xi^{n} \exp \left(-\mu A^{\beta} T w n \epsilon^{-\beta}\right) \\
& \leq \exp \left(n\left(\log (\xi)-K / \epsilon^{\beta}\right)\right)
\end{aligned}
$$

where in the second inequality we used the following result of Corollary 3.1

$$
\mathbf{E}^{0}\left[\mathcal{H}_{0}^{\text {out }, n ; W=0}(0)\right]=h^{\text {out }, n ; W=0}=h^{\text {in }, k, W=0} \leq \xi^{k}
$$

and where $K$ is a positive constant. This shows (24) for $\epsilon$ small enough, and thus concludes the proof.

Proof. (of Corollary 3.5) Without loss of generality assume $x=0$. We use the left inequality in (6), (14) and the inclusion (16) to obtain

$$
P_{X(0), X(y)}(0) \geq L_{X(0)}(0) \geq \mathcal{T}_{X(0)}(0) \geq \widehat{\mathcal{T}}_{X(0)}(0)
$$

and in consequence

$$
p(0, y, \Phi) \geq \mathbf{E}\left[\widehat{\mathcal{T}}_{X(0)}(0) \mid \Phi\right]
$$

Using the isotropy and the strong Markov property of the Poisson p.p.

$$
\left.\mathbf{E}\left[\widehat{\mathcal{T}}_{X(0)}(0) \mid \Phi\right]=\mathbf{E}^{0}\left[\widehat{\mathcal{T}}_{0}(0)|\Phi|_{\bar{B}}\right)\right]
$$


$\left.\left.\Phi\right|_{\bar{B}}\right)$ is the restriction of $\Phi$ to the complement of the open ball $B=B_{(0, R)}(R)$, centered at $(0, R)$ of radius $R \geq 0$, where $R$ is r.v. independent of $\Phi$ and having for density

$$
\frac{\mathrm{d} \theta}{2 \pi} 2 \pi \lambda r \exp \left(-\lambda \pi r^{2}\right)
$$

But since we consider here the SNR graph $\widehat{\mathbb{G}}$

$$
\left.\mathbf{E}^{0}\left[\widehat{\mathcal{T}}_{0}(0)|\Phi|_{\bar{B}}\right)\right] \geq \mathbf{E}^{0}\left[\widehat{\mathcal{T}}_{0}(0) \mid \Phi\right]
$$

The result follows now from Lemma 4.

\section{SINR space-time graph and routing}

Let us now translate our results regarding the SINR graph into properties of routing in ad-hoc networks.

Firstly, it makes sense to assume that any routing algorithm builds paths on $\mathbb{G}$. This takes two key phenomena into account: contention for channel (nodes have to wait for some particular time slots to transmit a packet) and collisions (lack of capture due to insufficient SINR).

Our time constant gives bounds on the delays that can be attained in the ad-hoc network by any routing algorithms. Of course, realistic routing policies cannot use information about future channel conditions. In the case of Poisson p.p. there is hence no routing algorithm with a finite time constant. The existence of such an algorithm in the case of the Poisson+Grid p.p. remains an open question. In the Poisson p.p. case; one can ask about the exact asymptotics of the optimal delay (we know it is not linear) and of the delay realizable by some non-anticipating algorithm.

Let us discuss now the relation of our results to those obtained in $[12,15]$. In these papers the so called delay-tolerant networks are considered and modeled by a spatial SINR or signal-to-noise ratio (SNR) graph with no time dimension. In these models, the time constant (defined there as the asymptotic ratio of the graph distance to the Euclidean distance) is announced to be finite, even in the pure Poisson case. The reason for the different performance of these models lays in the fact that they do not take the time required for a successful transmission from a given node in the evaluation 
of the end-to-end delay. The heavy-tailness of this time (which follows from that of the exit time (cf. Proposition 3.1) makes the time constant infinite in the space-time Poisson scenario. The reason for the heavy-tailness of the successful transmission time is linked to the so called "RESTART" algorithm (see e.g. [17, 18, 1, 16]). In our case the spatial irregularities in the ad-hoc network play a role similar to that of the file size variability in the RESTART scenario.

\section{References}

[1] S. Asmussen, P. Fiorini, L. Lipsky, T. Rolski, and R. Sheahan. Asymptotic behavior of total times for jobs that must start over if a failure occurs. Mathematics of Operations Research, 33(4):932-944, 2008.

[2] F. Baccelli, B. Blaszczyszyn, and P. Mühlethaler. An Aloha protocol for multihop mobile wireless networks. In Proceedings of the Allerton Conference, Urbana Champaign, Illinois, November 2003. and IEEE Transactions on Information Theory, 52(2):421-436, 2006.

[3] F. Baccelli, B. Blaszczyszyn, and P. Muhlethaler. An aloha protocol for multihop mobile wireless networks. IEEE Transactions on Information Theory, 52(2):421436, 2006.

[4] F. Baccelli, B. Błaszczyszyn, and P. Mühlethaler. Stochastic analysis of spatial and opportunistic Aloha. IEEE JSAC, special issue on Stochastic Geometry and Random Graphs for Wireless Networks, 27:1109-1119, 2009.

[5] F. Baccelli, B. Błaszczyszyn, and P. Mühlethaler. Time-space opportunistic routing in wireless ad hoc networks, algorithms and performance. the Computer Journal, 2009. to appear.

[6] F. Baccelli and C. Bordenave. The radial spanning tree of a poisson point process. Annals of Applied Probab., 17(1):305-359, 2007.

[7] Charles Bordenave. Navigation on a poisson point process. Ann. Appl. Probab., 18:708-746, 2008. 
[8] Simon Broadbent and John Hammersley. Percolation processes I. crystals and mazes. Proc. Camb. Phil. Soc, 53:629-641, 1957.

[9] D. J. Daley and D. Vere-Jones. An Introduction to the Theory of Point Processes, vol. II. Springer, New York, 2008.

[10] O Dousse, F. Baccelli, and P Thiran. Impact of interferences on connectivity in ad-hoc networks. IEEE/ACM Trans. Networking, 13:425-543, 2005.

[11] O. Dousse, M. Franceschetti, N. Macris, R. Meester, and P. Thiran. Percolation in the signal to interference ratio graph. Journal of Applied Probability, 43(2):552$562,2006$.

[12] R. K. Ganti and M. Haenggi. Bounds on information propagation delay in interference-limited ALOHA networks. In Proc. of Workshop on Spatial Stochastic Models for Wireless Networks, 2009.

[13] E. N. Gilbert. Random plane networks. SIAM J., 9:533-543, 1961.

[14] C. D. Howard and C. M. Newman. Euclidean models of first-passage percolation. Probab. Theory Relat. Fields, 108:153-170, 1997.

[15] P. Jacquet, B. Mans, P. Mühlethaler, and G. Rodolakis. Opportunistic routing in wireless ad hoc networks: Upper bounds for the packet propagation speed. IEEE JSAC, special issue on Stochastic Geometry and Random Graphs for Wireless Networks, 27:1192-1202, 2009.

[16] P. R. Jelenkovic and J. Tan. Stability of finite population aloha with variable packets. Technical Report arXiv:0902.4481v2, 2009.

[17] P.R. Jelenković and J. Tan. Can retransmissions of superexponential documents cause subexponential delays? In Proc. of INFOCOM, Anchorage, AL, USA, 2007. IEEE.

[18] P.R. Jelenković and J. Tan. Is aloha causing power law delays? In L. Mason, T. Drwiega, and J. Yan, editors, Managing Traffic Performance in Converged Networks. Springer, Berlin, 2007. 
[19] J.F.C. Kingman. Subadditive ergodic theory. Annals of Probab., 1:883-899, 1973.

[20] J.M. Kleinberg. The small-world phenomenon: an algorithmic perspective. In Proc. 32nd Annual ACM Symposium on the Theory of Computing, pages 163$170,2000$.

[21] L.P.R. Pimentel. The time constant and critical probabilities in percolation models. Elect. Comm. in Probab., 11:160-168, 2006.

[22] Ch. Pugh and M. Shub. Ergodic elements of ergodic actions. Compositio Mathematica, 23(1):115-122, 1971.

[23] D. Tse and P. Viswanath. Foundamentals of Wireless Communication. Cambridge University Press, 2005.

[24] M. Q. Vahidi-Asl and J. C. Wierman. First-passage percolation on the Voronoi tessellation and Delaunay trangulation. In M. Karośki, J. Jaworski, and A. Ruciński, editors, Random Graphs'87; Based on Proceedings of the 3rd International Seminar on Random Graphs and Probabilistic Methods in Combinatorics, June 27 - July 3, pages 341-359. John Wiley \& sons, Chichester, 1990. 